\title{
Temps évolutionniste et temps révolutionnaire (Michelet, Flaubert, Zola)
}

\section{Juliette Azoulai}

\section{(2) OpenEdition \\ 1 Journals}

Édition électronique

URL : http://journals.openedition.org/aes/907

DOI : $10.4000 /$ aes.907

ISSN : 2258-093X

Éditeur

Laboratoire LISAA

Référence électronique

Juliette Azoulai, «Temps évolutionniste et temps révolutionnaire (Michelet, Flaubert, Zola) », Arts et Savoirs [En ligne], 7| 2016, mis en ligne le 06 décembre 2016, consulté le 01 mai 2019. URL : http:// journals.openedition.org/aes/907; DOI : 10.4000/aes.907

Ce document a été généré automatiquement le 1 mai 2019.

Centre de recherche LISAA (Littératures SAvoirs et Arts) 


\title{
Temps évolutionniste et temps
} révolutionnaire (Michelet, Flaubert, Zola)

\author{
Juliette Azoulai
}

1 Employé à l'origine dans un contexte astronomique, pour désigner le retour d'un astre après le parcours de son orbe, le mot « révolution » est progressivement passé de son sens spatial à un sens chronologique - signifiant alors la fin d'un temps (ce qui est révolu) -, puis à un sens historique, pour exprimer les mutations des gouvernements et des sociétés. Du ciel à la terre, de l'espace au temps, du calme à la violence, le terme «révolution » a connu de multiples vicissitudes ${ }^{1}$.

2 Le parcours mouvementé de ce concept entrelace également dès le XVIII ${ }^{\mathrm{e}}$ siècle l'histoire et l'histoire naturelle, temps humain et temps géologique. Buffon déjà avant Cuvier utilise le terme dans son Histoire et théorie de la Terre de 1749 pour désigner les altérations de la surface terrestre responsables de la formation de fossiles et de sédiments ${ }^{2}$. Le XVIII ${ }^{\mathrm{e}}$ siècle est ainsi le moment d'un constat inquiétant : il n'y a plus lieu d'opposer la constance divine de la création à l'inconstance des sociétés humaines; l'œuvre divine, la Terre, connait elle aussi des révolutions.

C'est avec la paléontologie de Cuvier que va s'affirmer le concept de « révolution du globe »; l'existence de fossiles d'espèces disparues prouve, écrit Cuvier, l'existence d'un "monde antérieur au nôtre, détruit par une catastrophe quelconque »; et la succession des espèces est rythmée par une série de cataclysmes. Le catastrophisme, selon le mot inventé par Whewell ${ }^{4}$, instaure ainsi un paradigme de discontinuité pour penser le temps géologique et biologique. Or, à travers le terme de "révolution», les théoriciens catastrophistes du début du XIX ${ }^{\mathrm{e}}$ siècle réactivent un imaginaire historique tout récent : celui de la période révolutionnaire, véritable soubresaut de l'histoire, mutation brusque et traumatisante, rupture temporelle entre un avant et un après.

4 Mais le XIX ${ }^{\mathrm{e}}$ siècle voit également se développer dans les sciences naturelles un paradigme de la continuité, qui de Lamarck jusqu'à Haeckel, en passant par Darwin et Spencer, va 
progressivement s'imposer sous le nom d'évolutionnisme, et essaimer dans le champ de la pensée historique.

5 C'est cette interaction entre deux conceptions concurrentes du temps - la temporalité évolutionniste et la temporalité révolutionnaire, toutes deux opératoires dans le double domaine de l'histoire et de la biologie - que nous proposons d'étudier ici. L'étude de textes français du second dix-neuvième siècle, tirés de Michelet, de Flaubert et de Zola, nous permettra de cerner les réflexions proposées par ces écrivains sur le caractère hybride des concepts d'évolution et de révolution et sur l'intersection qu'ils impliquent entre nature et culture.

\section{Michelet et les révolutions naturelles}

Dans La Montagne, publiée en 1868, Michelet décèle une concordance entre les théories des géologues et le contexte historique dans lequel elles apparaissent. Il retrace ainsi la généalogie du catastrophisme dans l'histoire chaotique de la société française; quant à l'uniformitarisme, qui récuse l'idée des bouleversements soudains et universels et qui explique l'histoire de la Terre à partir de l'action continue et à intensité constante des mêmes facteurs naturels, Michelet en voit l'origine dans le climat stable et apaisé de l'Angleterre. L'intellect scientifique, lorsqu'il s'applique à étudier les forces naturelles, s'ancre d'abord et avant tout, selon Michelet, dans le terreau d'une histoire collective. Les sciences de la nature sont aussi des faits de culture :

Ceux qui avaient assisté à l'éruption terrible du volcan révolutionnaire, aux catastrophes des grandes guerres, aux soulèvements nationaux de 1815, à l'immense tremblement de terre où l'Empire fut abîmé, - ceux-là ne virent nulle autre chose dans les origines du globe. Ils observaient avec les yeux, les mêmes yeux qui voyaient ces événements politiques. [...] Voilà la géologie qu'on faisait sur le continent, sur la terre des révolutions. Mais l'immobile Angleterre qui n'avait pas eu chez elle nos grandes secousses sociales, jugeait le globe autrement. Qu'avait-elle vu dans son sein? Une constitution progressive qui s'est faite peu à peu sans grand changement - un gouvernement d'équilibre qui change infiniment peu - une nouveauté, il est vrai, l'Angleterre industrielle qui assez rapidement, mais sans crise, sans combat, s'est peu à peu élevée. [...] Au fort de nos soulèvements, à peu près vers 1830, quand Buch, Élie de Beaumont semblaient régner, s'éleva une voix grave, la géologie de Lyell. Livre puissant, ingénieux, où pour la première fois la terre figure comme une ouvrière qui, d'un labeur pacifique, incessant, et sans secousse, se manufacture elle-même. ${ }^{5}$

7 Ainsi évoquant les théories d'Élie de Beaumont à propos du soulèvement des montagnes, théories qui se situent sous le patronage du système de révolutions de Cuvier, Michelet perçoit dans ces théories le reflet des événements politiques du tout premier XIX ${ }^{e}$ siècle : ces événements sont évoqués à travers des métaphores géologiques («volcan révolutionnaire ", "tremblement de terre où l'Empire fut abîmé »), ou à travers des syllepses, c'est-à-dire l'utilisation de termes en deux sens différents, applicables aussi bien aux mouvements sociaux qu'aux mouvements de la surface terrestre: «catastrophes des grandes guerres» (ce qui rappelle le catastrophisme géologique), "soulèvements nationaux de 1815 » (ce qui rappelle la théorie du soulèvement des montagnes de Beaumont), «soulèvements de 1830 ». De même la théorie uniformitarienne de Lyell en Angleterre est évoquée en termes politiques (un "gouvernement d'équilibre ») et en termes économiques, à travers la métaphore industrielle qui renvoie là encore la situation anglaise (la Terre est comparée à une ouvrière qui se manufacture elle-même). 
Ailleurs dans la même œuvre, Michelet évoquera à propos d'un géologue français son aptitude à ausculter les « 93 de la terre $»^{6}$, métaphore historique frappante pour parler du temps géologique.

Michelet va ainsi jusqu'à esquisser une phénoménologie de la connaissance: les sciences naturelles s'enracinent dans la vie quotidienne et plus précisément dans un vivre ensemble qui façonne la Weltanschauung des scientifiques. Le rapport immédiat au temps contemporain induit une certaine conception du temps des origines; tout est une affaire de regard et donc d'incarnation dans un hic et nunc: « Ils observaient avec les yeux, les mêmes yeux qui voyaient ces événements politiques $»^{7}$.

Cette double réfraction des théories géologiques dans l'histoire et de l'histoire dans les théories géologiques n'exclut pas un certain nombre d'exceptions; Michelet évoque ainsi Constant Prévost, géologue actualiste ${ }^{8}$, ou encore Lamarck, comme exemples de naturalistes français qui seraient plus proches des conceptions britanniques et finalement de l'esprit flegmatique anglais : «Lamarck avait, dès 1800, dit que la lente douceur des procédés de la Nature, que l'influence des milieux, surtout l'infini du temps, suffirait à tout expliquer, sans violence, sans coup d'État pour créer ou pour détruire. $»^{9}$

Surtout, Michelet voit dans cette "géologie des transformations paisibles » la trace de l'influence des théories transformistes en matière de zoologie: l'uniformitarisme géologique de Lyell trouve un « secours fraternel » dans les travaux des « grands maîtres en métamorphoses, notre Geoffroy Saint-Hilaire, Gœthe, Oken, Owen, Darwin ». Ainsi la représentation d'un temps géologique uniforme et continu est-elle liée aux récentes découvertes de la biologie, de sorte que cette « nouvelle géologie » est, dit-il, «l'étude des mouvements, des changements que fait en lui ce bel animal, la Terre $»^{10}$. Le temps géologique de Lyell correspond non seulement au temps continu et pacifique de l'histoire anglaise mais aussi et plus largement à la temporalité évolutionniste du vivant et de l'animal.

11 À la fin de sa démonstration Michelet en appelle à une synthèse du catastrophisme et de l'uniformitarisme, c'est-à-dire de l'esprit français et de l'esprit anglais, à travers une alliance des concepts d'évolution et de révolution géologiques. D'ailleurs, lorsque sept ans plus tôt Michelet publiait La Mer, en s'inspirant des théories de l'évolutionnisme prédarwinien ${ }^{11}$, il représentait déjà le temps de l'évolution biologique comme un temps non entièrement homogène, connaissant lui aussi des révolutions. Ainsi il décrivait l'apparition du poisson, comme une " révolution hardie $»^{12}$ par rapport à ses ancêtres les crustacés : «Le crustacé s'entourait comme d'un squelette extérieur. Le poisson se le fait au centre, en son intime intérieur, sur l'axe où les nerfs, les muscles, tout organe viendra s'attacher. $»^{13}$ Ce texte est vraisemblablement inspiré par la lecture de Geoffroy SaintHilaire $^{14}$ qui, en comparant l'anatomie du carrelet à celle du homard, met au jour une homologie entre les invertébrés et les vertébrés ${ }^{15}$, qui lui permet d'étayer l'hypothèse selon laquelle "tout animal vit en dehors ou en dedans de sa colonne vertébrale ${ }^{16}$. Il n'en reste pas moins que chez Michelet ce passage de l'exosquelette à l'endosquelette, cette révolution organique, est décrit comme une véritable métamorphose. Or l'idée de métamorphose s'oppose ici à l'esprit du transformisme, dans la mesure où le transformisme n'admet «dans l'histoire d'une forme survivante [...] aucune rupture, aucune révision brusque de son plan d'organisation [...], aucune innovation radicale. $»^{17}$ La métamorphose micheletienne rend au contraire le poisson méconnaissable par rapport à la forme de son ancêtre le crustacé, et cette fracture du lien généalogique, cette altérité fondamentale, se traduit dans le texte de Michelet par le surgissement du rire : 
Le crustacé dut en rire, quand il vit la première fois un être mou, gros, trapu (les poissons de la mer des Indes), qui, s'essayant, glissait, coulait, sans coquille, armure, ni défense; n'ayant sa force qu'au dedans, protégé uniquement par sa fluidité gluante, par le mucus exubérant qui l'entoure, et qui, peu à peu, se fixe en écailles élastiques. ${ }^{18}$

12 Ainsi les êtres passéistes, incarnés par le crustacé, rient des révolutionnaires, ici le poisson, capables d'entrer résolument dans un avenir métamorphosé. Mais le poisson, lui, qui est un "être de pure audace » et de "mouvement», méprise les crustacés qu'il considère comme "tardigrades $»^{19}$ - lexique zoologique, désignant les animaux qui marchent lentement, et qui exprime aussi bien, au sens figuré et politique, une mentalité rétrograde. Le progrès de la Nature (rappelons que Michelet pense alors l'échelle des êtres sur un mode téléologique) est analogue au progrès historique et fonctionne par brusques accélérations du devenir (les révolutions), précédées et suivies de lentes transformations : ainsi le poisson est d'abord un mollusque nu et vulnérable avant de se constituer «peu à peu » des écailles. Michelet combine à l'idée évolutionniste ce qu'on pourrait appeler une forme de révolutionnisme, si l'on entend par là l'application à la biologie du concept historique de révolution.

\section{Flaubert et l'évolution socio-historique}

13 Au contraire, Flaubert s'efforcera, à la fin des années 1870, dans sa correspondance, de déconstruire l'idée politique de révolution, en proposant une lecture biologique de l'histoire, soit une lecture évolutionnaire des événements politiques, si l'on entend par ce terme un point de vue radicalement opposé à celui des révolutionnaires. Ainsi écrit-il en 1879 : «La théorie de l'évolution nous a rendu un fier service! Appliquée à l'Histoire elle met à néant les rêves sociaux. $»^{20}$

14 Ce point de vue va à l'encontre de ce que Flaubert épingle comme une doxa de l'époque : à savoir que les partisans des théories évolutionnistes en matière de sciences naturelles seraient de terribles matérialistes et donc des éléments perturbateurs de l'ordre social. Dans Bouvard et Pécuchet par exemple, les deux héros sont très bien vus des notables de Chavignolles et en particulier du prêtre, tant qu'ils adhèrent à la géologie catastrophiste de Cuvier, car celle-ci confirme le Déluge ${ }^{21}$. Mais lorsqu'ils font des lectures transformistes, qu'ils lisent Lamarck et Geoffroy Saint Hilaire, et qu'ils entreprennent alors de mettre en cause l'autorité des Écritures au nom de l'évolutionnisme, le capitaine Heurtaux leur crie : « Vous êtes des révolutionnaires! » ${ }^{22}$ Flaubert met donc en lumière le nœud inextricable qui mêle sciences naturelles, idéologie politique et religion. Les révolutions cuviériennes du globe étaient compatibles avec la représentation d'une transcendance divine, à la fois créatrice et destructrice, puisque les cataclysmes universels sont finalement des événements tellement extraordinaires qu'ils semblent quasi surnaturels; le catastrophisme correspond donc à un point de vue politique conservateur. Au contraire, s'opposer aux révolutions cuviériennes au nom de l'évolutionnisme scientifique, c'est contester la représentation religieuse du temps et, en s'attaquant à la religion, c'est être révolutionnaire.

Dans le Dictionnaire des idées reçues, Flaubert révèle le même type de concrétion politicoscientifique à propos de la question des générations spontanées : « GÉNÉRATION SPONTANÉE : idée de socialiste. ${ }^{23}$ La thèse de la génération spontanée, défendue par Félix-Archimède Pouchet, est contredite au début des années 1860 par Pasteur, qui s'érige alors en garant 
de l'orthodoxie religieuse, contre les matérialistes de tout bord qui voudraient accorder à la matière seule le pouvoir de créer la vie. Ainsi la génération spontanée, qui postule l'apparition de la vie sur Terre à partir de la matière inerte apparait comme la pierre angulaire d'un édifice évolutionniste total, réunissant géologie et biologie à l'intérieur d'une même histoire de la Nature indépendante de Dieu. Or cet affranchissement radical vis-à-vis de l'idée d'un Dieu créateur en confinant à une forme d'athéisme serait ainsi le propre des contestataires socialistes. Là encore, selon les idées reçues de l'époque, le jusqu'auboutisme évolutionniste rejoindrait l'esprit de subversion révolutionnaire.

Mais Flaubert stigmatise cet amalgame précisément parce que la relation entre évolution et révolution est selon lui, en réalité, une relation d'antagonisme et que l'évolutionnisme bien compris réduit à néant l'idéal révolutionnaire. C'est tout le sens du débat que Flaubert engage avec Maxime du Camp au sujet de l'idéologie communarde. À la parution du dernier tome des Convulsions de Paris, Flaubert écrit à son ami pour le complimenter mais aussi (et surtout?) pour en contester certains passages, en particulier l'analyse que fait Du Camp des effets néfastes du darwinisme sur les insurgés de la Commune :

Il y a une page que je voudrais effacer de ton volume, la page 244 : Les côtés dangereux de la théorie de Darwin! Est-ce sérieux ? Et tu avoues toi-même qu'elle a agi sur les communeux un peu à leur insu. Je crois même qu'ils l'ignoraient complètement [...]. C'est l'Économie politique (ou mieux « l'infâme » Malthus) qui a inspiré Darwin. Il serait temps que la sociologie s'inspirât de lui. C'est d'ailleurs ce qu'elle fait en Angleterre. Quand ces idées-là seront descendues dans les masses, il n'y aura plus de révolutions parce qu'on sera convaincu que « Natura non facit saltus $! »^{24}$ l'histoire selon Du Camp connaît des secousses, ce qu'il appelle des «crises d'épilepsie sociale $\aleph^{25}$, comme l'insurrection communarde. Du Camp lui-même biologise donc l'histoire, à travers l'image d'une pathologie sociale, productrice d'une temporalité spasmodique et saccadée. Or, pour Du Camp, la loi d'évolution darwinienne, par «ses côtés dangereux", alimente cette folie des révoltes sociales: la Commune serait souterrainement un produit de l'idéologie darwinienne. Et il cite notamment à l'appui de cette idée l'affaire Lebiez et Barré, qui avait défrayé la chronique en 1878 : deux étudiants désargentés, issus de la province et faisant leurs études à Paris, qui ont assassiné une vieille laitière pour lui voler son argent ; or l'un d'entre eux s'était fait remarquer peu de temps avant son arrestation par une conférence au quartier latin sur le principe de la lutte darwinienne pour la vie. Cette affaire inspirera à Barrès la thèse de son roman Les Déracinés (1897), dont le personnage principal est également, comme on le dit à l'époque, un struggleforlifeur ${ }^{26}$; l'histoire de Lebiez et Barré est donc un point de cristallisation des hantises de la bourgeoisie conservatrice par rapport au darwinisme. La théorie darwinienne est alors accusée de propager dans la société le principe d'une loi de la jungle ${ }^{27}$ susceptible d'aboutir au renversement de l'édifice social.

Or Flaubert s'insurge contre une telle assimilation de l'évolutionnisme à l'esprit de sédition. Pour lui, la loi de sélection darwinienne est issue des principes de Malthus ; c'est donc l'économie qui donne ses règles à la biologie évolutionniste et si en retour la biologie doit inspirer une politique, ce ne sera jamais dans le sens d'un quelconque égalitarisme. À travers ce débat entre Flaubert et Du Camp transparaissent les particularités de la réception de Darwin au XIX ${ }^{e}$ siècle : la loi d'évolution est en effet appréhendée à travers le filtre de la sociologie spencérienne. Le darwinisme, comme le souligne Yvette Conry ${ }^{28}$, s'introduit en France sous la forme d'un mélange de 
transformisme et d'idéologies sociales et politiques. La pensée darwinienne de la sélection est réduite au noyau malthusien de la concurrence interindividuelle; et appliquée aux sociétés humaines, elle sert une idéologie ultralibérale.

Flaubert s'inscrit donc dans cette lecture social-darwinienne, prônant une «idéologie de la radicale immanence $»^{29}$, où les sociétés sont conçues comme des organismes capables de s'autoréguler naturellement. Dès lors toute tentative de modifier ou de transgresser l'ordre social est-elle vouée à l'échec. L'histoire des sociétés, comme l'histoire de la Nature, avance pas à pas selon un principe gradualiste : Natura non facit saltus. La nature ne fait pas de saut. Cet axiome linnéen, repris par Darwin, implique ainsi une forme d'immobilisme et d'attentisme politiques: il n'y a rien à faire, puisque tout se fait progressivement. Pas de révolution possible puisque tout n'est qu'évolution.

Ce débat entre Flaubert et Du Camp sur la portée socio-politique du darwinisme avait déjà opposé les deux naturalistes allemands Virchow et Hæckel au congrès de Munich en 1877. Tandis que Virchow accusait le darwinisme d'être le cheval de Troie du socialisme, Hæckel ripostait dans ses Preuves du transformisme que la doctrine de l'évolution est un " contrepoison contre les absurdes utopies égalitaires des socialistes $»^{30}$. Flaubert se range par conséquent du côté de Hæckel, qu'il lit avec grand intérêt dans les années 1870 : son Histoire de la création lui semble un livre "plein de faits et d'idées " ${ }^{31}$, «qui fait joliment rêver » $^{32}$, où le darwinisme est "plus clairement exposé que dans les livres de Darwin, même $»^{33}$.

21 L'écrivain trouve dans le social-darwinisme de Hæckel et de Spencer une confirmation de ses positions sociales élitistes et de ses vues politiques réactionnaires, mais il y trouve surtout une vision continuiste du temps historique, qui lui apparait comme un désenchantement salutaire de l'idée de révolution. Ce que Flaubert reproche aux socialistes révolutionnaires, c'est non seulement le contenu de leur utopie, l'égalitarisme, mais aussi la forme de sa réalisation, à savoir la révolution censée changer brusquement et volontairement le cours de l'histoire. Pour Flaubert, l'idéal révolutionnaire relève de l'illusion mystique ou messianique : « La Magie croit aux transformations immédiates par la vertu des formules, absolument comme le Socialisme. Ni l'une ni l'autre ne tiennent compte du temps et de l'évolution fatale des choses. $»^{34}$

En croyant que l'idéal a un pouvoir de création ex nihilo, l'idée révolutionnaire reconduit le transcendantalisme chrétien, et relève de la croyance au miracle. On peut dès lors comprendre la notice «GÉNÉRATION SPONTANÉE » du Dictionnaire des idées reçues d'une tout autre manière, en y reconnaissant non plus la voix de l'adversaire bourgeois, mais la voix de l'écrivain lui-même. Si la génération spontanée est une idée de socialiste, c'est peut-être qu'il faut la transposer, du domaine biologique qui est originellement le sien, au domaine de l'histoire: or, admettre l'apparition d'une forme de vie «sans parents » pour la transmettre (puisque telle est la définition de la génération spontanée en biologie), c'est admettre l'existence d'un événement dépourvu d'antécédents, c'est donc croire aux bonds de l'histoire, c'est-à-dire aux révolutions, c'est croire que la Nature et l'Histoire font des sauts. Les socialistes, selon Flaubert, croient à la génération spontanée d'une société égalitaire. 


\section{Zola, la complémentarité entre évolution et révolution}

Zola met lui aussi en lumière dans Les Rougon-Macquart le lien étroit qu'entretiennent conviction socialiste et déraison religieuse: dans Germinal les discours enthousiastes d'Étienne Lantier et l'exaltation qu'ils suscitent chez les mineurs rappellent la ferveur et l'idéal du christianisme primitif, et sont présentés comme des mystifications: ce qu'Étienne croit apercevoir et ce qu'il fait entrevoir à ses auditeurs, c'est l'illusion « d'une société nouvelle poussa[n]t en un jour, ainsi que dans les songes $\aleph^{35}$. Pourtant cette critique de l'idéalisme révolutionnaire ne s'appuie pas, comme chez Flaubert, sur la sociobiologie évolutionniste. Zola, dans Germinal, souligne plutôt les rapports de tension et de complémentarité qui unissent l'idée de révolution à celle d'évolution, les forces historiques aux forces biologiques et pose ainsi la question de la discordance (ou de possible concordance) entre la temporalité sociale et la temporalité naturelle. Expliquant le choix de son titre, Zola affirme avoir eu la vision d'« un avril révolutionnaire, une envolée de la société caduque dans le printemps $\aleph^{36}$. L'écrivain superpose alors deux représentations contradictoires du temps : la secousse révolutionnaire, qui marque une rupture entre un avant et un après; et la continuité des saisons, au sein de laquelle le printemps succède naturellement à l'hiver, la régénérescence à la caducité.

C'est en premier lieu dans les discussions entre les personnages de Germinal qu'apparaît une confrontation entre le temps révolutionnaire et le temps évolutionniste. Étienne Lantier, plus instruit que les autres mineurs, est d'abord le personnage en qui germe un esprit de révolte et il penche alors pour le socialisme historico-scientifique, ce qui suscite les moqueries de son camarade anarchiste, Souvarine :

- Des bêtises ! répéta Souvarine. Votre Karl Marx en est encore à vouloir laisser agir les forces naturelles. [...] Fichez-moi donc la paix, avec votre évolution! Allumez le feu aux quatre coins des villes, fauchez les peuples, rasez tout, et quand il ne restera plus rien de ce monde pourri, peut-être en repoussera-t-il un meilleur. ${ }^{37}$

Pour le penseur d'une révolution radicale qu'est Souvarine, le marxisme est une pensée trop évolutionniste et pas assez révolutionnaire; Souvarine stigmatise alors le déterminisme historique de Marx qu'il rapproche du darwinisme : «tes savants sont des lâches avec leur évolution $\aleph^{38}$. Il est vrai que le marxisme substitue aux représentations idéalistes de l'histoire une connaissance critique du mouvement historique, qui peut apparaître comme "une théorie de l'évolution des systèmes politiques sur la base des changements survenus au sein du système productif ${ }^{39}$. Zola s'inspire alors d'éléments puisés dans la synthèse d'Émile de Laveleye sur le socialisme contemporain ${ }^{40}$. Laveleye dépeint ainsi le marxisme comme un "fatalisme $»^{41}$ et cite à l'appui de son propos une phrase du Capital où Marx naturalise le processus historique, à travers des métaphores biologiques :

Lors même qu'une société est arrivée à découvrir la voie de la loi naturelle qui préside à son mouvement, elle ne peut ni dépasser d'un saut, ni abolir par décrets les phases de son développement naturel, mais elle peut abréger la période de gestation et adoucir les maux de leur enfantement. ${ }^{42}$ Autrement dit, l'Histoire pour Marx, comme la Nature pour Darwin, ne fait pas de saut. L'anarchiste Souvarine dénonce ainsi la collusion du marxisme et de l'évolutionnisme, que Laveleye critique lui-même, dans une tout autre perspective, au nom du socialisme chrétien. Laveleye décrit dans sa préface "l'étrange aveuglement» des socialistes 
adoptant « les théories darwiniennes qui condamnent leurs revendications égalitaires »" De même, Souvarine s'emportera contre «la bêtise des socialistes qui acceptent Darwin, cet apôtre de l'inégalité scientifique, dont la fameuse sélection n'était bonne que pour des philosophes aristocrates $»^{44}$. La théorie de la sélection darwinienne n'est bonne qu'à justifier l'économie politique libérale du "laissez-faire, laissez-passer » et de la libre concurrence, et ne saurait étayer aucun projet authentiquement socialiste : le darwinisme n'aboutit qu'à une naturalisation de l'inégalitarisme social. Étienne lui-même, quoique lecteur de Darwin, s'opposera à une forme de socialisme qu'il jugera également trop évolutionniste, c'est le possibilisme de Rasseneur, qui affirme «la nécessité de laisser à l'évolution sociale le temps de s'accomplir " $^{45}$.

Au centre du positionnement idéologique des personnages se situe donc la question du lien entre la biologie évolutionniste et l'histoire socialiste révolutionnaire. De fait, Germinal pointe de manière récurrente l'ambiguïté et la complexité des rapports entre ces deux grandes pensées du XIX ${ }^{e}$ siècle : marxisme et darwininisme. On sait que peu de temps après la parution en anglais de L'Origine des espèces, Marx affirmait dans une lettre à Lassalle (16 janvier 1861) que le livre de Darwin peut être conçu comme «base de la lutte historique des classes $»^{46}$ - le struggle for life biologique permettant d'offrir un fondement naturel au principe historique de la lutte des classes. Marx et Darwin convergent de la même manière dans l'esprit d'Étienne Lantier, qui se fait « une idée révolutionnaire du combat pour l'existence, les maigres mangeant les gras, le peuple fort dévorant la blême bourgeoisie $»^{47}$. Mais en 1862 Marx dénoncera chez Darwin l'application à la biologie des théories de l'économie bourgeoise et du malthusianisme; et Lafargue, le gendre de Karl Marx, écrira que «les darwiniens ne font que rabâcher naturalistement la leçon des économistes $\aleph^{48}$. Le discours marxiste entretient une relation ambivalente à la loi d'évolution darwinienne, tantôt vantée comme arme révolutionnaire, tantôt vilipendée comme instrument de légitimation du statu quo inégalitaire; et le roman de Zola reflète cette ambivalence.

Par ailleurs, la conception anarchiste de la révolution, en tant qu'elle implique la destruction pure et simple de la société actuelle, apparaît au héros contre-nature, parce qu'elle coupe définitivement le lien entre le passé et l'avenir :

Et puis, il ne comprenait toujours pas, sa race se refusait au rêve sombre de cette extermination du monde, fauché comme un champ de seigle, à ras de terre. Ensuite, que ferait-on, comment repousseraient les peuples ? ${ }^{49}$

La métaphore agricole montre bien à quel point Étienne refuse de dissocier l'histoire de la nature, le rythme des sociétés de celui de la vie. Et en un sens, c'est le problème majeur autour duquel se construit le roman de Zola: comment trouver les moyens d'une réconciliation entre évolution et révolution, entre temps biologique et temps historique?

S'il semble difficile de la trouver au plan purement idéologique, le parcours d'Étienne nous montre, sur un plan cette fois proprement romanesque et dans le travail du style, la voie d'une réconciliation possible. La mise en roman de ce questionnement philosophique fournit alors le moyen de sortir de l'aporie théorique suivante : soit, comme Rasseneur, on "attend tous des faits, de l'évolution $"^{50}$ et rien ne change; soit, comme le veulent Étienne et Souvarine, on fait la révolution et alors le temps est hors de ses gonds et la table rase est une pure destruction sans perspective de renouveau. Il s'agit donc de penser une révolution qui ne soit pas contre-nature et qui ne soit pas non plus une adhésion aveugle au déterminisme historico-naturel. Or c'est ce que le roman parviendra 
à faire in fine, grâce au savant montage de la narration et grâce au réseau de métaphores qui unifie l'ensemble de l'œuvre.

Colette Becker voit dans le déroulement du roman un passage du « règne minéral » (celui de la mine) au «règne végétal» (celui de la germination), du «statique, défini une fois pour toutes" au «dynamique », de la «mort» à la "vie » ${ }^{51}$. L'histoire des mineurs de Montsou est celle d'une ouverture du monde mécanique, figé dans une éternelle répétition, sur le monde du vivant ou plutôt l'histoire d'une synchronisation de la temporalité des travailleurs et de la temporalité naturelle. En effet, si le rêve de la révolution anarchiste semble inhumain et dénaturé, l'inégalitarisme capitaliste l'est tout autant et ceci se marque en particulier dans le décalage entre le rythme de vie des mineurs et le rythme cyclique de la Nature. La diégèse de Germinal se déroule à peu près en l'espace d'un an, d'un mois de mars hivernal à un mois d'avril printanier. On y assiste donc au retour de deux printemps : or le premier printemps est un printemps manqué pour les travailleurs enfermés dans « l'éternel hiver du fond, au milieu de ces ténèbres humides que jamais ne dissipait aucun été $»^{52}$; et Zola poursuit : "Le printemps était venu. [...] Des herbes envahissaient le terri, des fleurs couvraient les prés, toute une vie germait, jaillissait de cette terre, pendant qu'il geignait sous elle, là-bas, de misère et de fatigue. $\aleph^{53}$ Discordance majeure entre la temporalité de la Nature et celle des travailleurs.

Les dernières phrases du roman montreront au contraire la coïncidence du printemps naturel et du printemps humain : la germination dans la nature est corrélée à la poussée d'hommes nouveaux au sein de la société. Mais cette mise en concordance des deux temporalités n'a pas pu se faire d'elle-même, spontanément; il a fallu que l'action révolutionnaire remette en marche le dynamisme de l'évolution, que la secousse violente de la révolte donne l'impulsion d'un progrès continu et graduel.

C'est ce que montre notamment le corps de Catherine Maheu, pétrifié au début du roman dans une éternelle enfance : à quinze ans, elle «attend encore la crise $»^{54}$ pubertaire. Cette désynchronisation entre le temps de l'ouvrière et le temps naturel du corps féminin est symptomatique de l'écart qui sépare le monde immobilisé des mineurs et le monde mouvant de la nature. Catherine Maheu finira par connaître la « crise » tant attendue, au moment même où elle s'éveillera à la rébellion collective et violente ; lorsque les mineurs grévistes s'opposent aux soldats, épisode qui sera le plus sanglant de toute la grève puisque l'armée finira par tirer sur la foule des mineurs, Catherine connaît une métamorphose intérieure, d'abord au plan psychologique, puisqu'elle se révolte pour la première fois. Une forme de rage « crève » en elle, véritable soubresaut psychique, auquel correspond simultanément un bouleversement physiologique, le déclenchement de ses premières règles : « c'était le flot de la puberté qui crevait enfin, dans la secousse de cette journée abominable $»^{55}$. C'est donc à la faveur d'une crise politique qu'a lieu la crise pubertaire. Le terme de crise, utilisé par Zola pour évoquer la puberté de Catherine, est ainsi exploité dans son double sens médical et politique, le corps individuel étant indissociable du corps social. Or la crise au sens médical, cette brusque transformation, est intimement liée à l'évolution générale de l'organisme. Le flot de la puberté qui crève dans le corps de Catherine, véritable révolution, est la condition d'une remise en route de l'évolution naturelle de l'organisme. Il devient dès lors possible de penser les notions d'évolution et de révolution non plus comme seulement antagonistes mais aussi comme complémentaires, la révolution permettant d'harmoniser le temps de la travailleuse et le temps de la femme, c'est-à-dire plus largement le temps social et le temps biologique ${ }^{56}$. 

des graines, leur lente poussée, le travail de l'enfantement), et en même temps sur le mode du bouleversement brusque : parvenue à maturité, la vie jaillit, les bourgeons crèvent, les graines humaines feront éclater la terre. Ces derniers mots du roman («faire éclater la terre ») témoignent d'une fidélité à l'exigence révolutionnaire, portée aussi bien par Souvarine, que par Rasseneur ou Étienne, et qui est exprimée à plusieurs reprises dans le roman à travers la formule: "Il faut que ça pète! $\aleph^{58}$ Tout l'objet du roman, d'après l'ébauche, est en effet de montrer (ce sont les mots de Zola) « la révolution sociale fatale $»^{59}$; or, cette fatalité est précisément inscrite dans le devenir naturel ; c'est parce que la nature est évolutive qu'il faut que ça pète, qu'il faut (impératif moral et loi ontologique) qu'il y ait révolution. La violence n'est donc pas exclue : l'image de «l'armée noire » et « vengeresse » qui surgira dans le siècle futur » ${ }^{60}$ est assez emblématique de cet imaginaire agonistique de la révolution, qui sera totalement absent du roman Travail de 1901, bien que ce dernier présente de nombreuses analogies avec Germinal. L'avènement de la Cité idéale, rompant avec l'exploitation de l'homme par l'homme, passe dans Travail par un schéma évolutionniste, qui exclut toute secousse révolutionnaire. Luc Froment, le héros de Travail, se fait alors l'apôtre de l'évolution, en prenant appui sur la doctrine de Fourier (c'est-à-dire sur la libre association): «Pas de révolution. Évolution pour Fourier $"^{61}$, note Zola dans son ébauche. Dès lors la mise en œuvre du projet social de Luc adopte le rythme biologique du progrès continu, sur le modèle de la croissance végétale. La ville nouvelle fondée par le personnage supplante peu à peu l'ancienne cité ouvrière : «il revenait toujours à ces vivantes maisons, que son souffle semblait faire sortir de terre et qu'il voyait réellement en marche, telle qu'une armée pacifique, partie pour ensemencer l'avenir sur les décombres du vieux Beauclair et de l'Abîme " ${ }^{62}$.

On remarque ici une métaphore très similaire à celle de la fin de Germinal ; mais les signes en sont inversés; au lieu d'une armée noire et vengeresse, c'est une armée pacifique qui sort de terre et promet un avenir radieux et apaisé. La construction progressive et sans heurt de la Cité nouvelle, est désignée à plusieurs reprises dans Travail à travers la métaphore de la poussée végétale mais qui a perdu sa dimension de violence, qui ne fait plus "éclater la terre»: "l'avenir est à nous, puisque notre ville pousse, pousse toujours " ${ }^{63}$. Le slogan " il faut que ça pète » de Germinal semble ainsi supplanté par une devise qui serait "il faut que ça pousse", à laquelle finissent par se rallier les révolutionnaires eux-mêmes.

La disparition de l'idée de révolution (encore présente en 1885 dans Germinal) au profit de l'évolution dans Travail, en 1901, trouve peut-être une explication dans le développement 
et la propagation des thèses anarchistes au cours des années 1890. En effet la pensée politique anarchiste cherche un fondement dans les sciences naturelles et en particulier dans l'évolutionnisme darwinien. Élisée Reclus tente ainsi en 1898 de rapprocher les notions d'ordinaire antagonistes d'évolution et de révolution dans une brochure intitulée L'évolution, la révolution et l'idéal anarchiste : l'évolution et la révolution sont envisagées par Reclus comme les "deux actes successifs d'un même phénomène " ${ }^{64}$ et pour expliquer cette solidarité des deux concepts il utilise l'analogie de la formation et de la disparition d'un lac:

Qu'un éboulis barre une rivière, les eaux s'amassent peu à peu au-dessus de l'obstacle, un lac se formera par une lente évolution, puis tout à coup une infiltration se produira dans la digue d'aval, la chute d'un caillou décidera du cataclysme, l'obstacle sera violemment emporté et le lac vidé redeviendra rivière : ainsi aura lieu une petite révolution terrestre. ${ }^{65}$

Travail en 1901, comme Paris en 1898, met en scène ce discours anarchiste qui tente d'assimiler le cataclysme, la révolution, au cours de la Nature en évolution. Ainsi Lange affirme-t-il dans Travail que « la catastrophe, le volcan [est] dans la nature ${ }^{66}$; Guillaume Froment dans Paris tenait un discours similaire :

C'est alors que, dans l'histoire du globe, puis dans celle des sociétés, il m'a fallu faire la place du volcan, le brusque cataclysme, la brusque éruption, qui a marqué chaque phase géologique, chaque période historique. On en arrive ainsi à constater que jamais un pas n'a été fait, un progrès accompli, sans l'aide d'épouvantables catastrophes. ${ }^{67}$

41 Cette instrumentalisation du catastrophisme géologique pour servir la cause terroriste est ainsi réprouvée par Zola, qui met cette thèse à distance en la plaçant dans la bouche du personnage. La pensée de la complémentarité entre évolution et révolution est désormais condamnée, alors qu'elle travaillait encore les métaphores de Germinal.

Mais Germinal parvenait encore d'une autre manière à lier révolution et évolution, à travers la structure narrative du roman d'éducation. À la fin du roman, écrit Zola dans son dossier préparatoire, Étienne s'en va, "son éducation socialiste achevée " ${ }^{68}$, et le texte final le montre "mûri " ${ }^{69}$, ayant pris conscience que «la violence peut-être ne hâtait pas les choses $»^{70}$ : il est passé de l'adolescence révoltée à la maturité révolutionnaire.

43 Le modèle du roman d'éducation, du Bildungsroman, auquel se rattache Germinal, nous montre ainsi un processus naturel et continu de formation d'un « soldat raisonneur de la révolution $»^{71}$. Malgré l'échec de la grève et le constat de la paralysie sociale (les mineurs reprennent le travail dans les mêmes conditions qu'auparavant), Zola nous montre, à travers son personnage, la révolution en marche, comme work in progress. En reprenant le modèle goethéen du Bildungsroman, Zola utilise un paradigme évolutif et naturel pour représenter la gestation d'une révolution sociale fatale. En effet, le Bildungsroman tel que le conçoit Goethe n'est pas sans lien avec la théorie de la métamorphose des plantes, dans les deux cas il s'agit de penser le développement de la plante ou de l'individu à travers un schéma progressif et unitaire. Ainsi le roman de formation zolien, en alliant la biographie à la chronique sociale, nous montre la formation organique (la Bildung) d'un révolutionnaire, Étienne, et d'une révolution (promise pour le $\mathrm{xx}^{\mathrm{e}}$ siècle).

Il serait donc réducteur de considérer que les métaphores naturelles chez Zola seraient au service de la défense d'un ordre social immuable ${ }^{72}$. Zola représente une nature en mouvement, en évolution, dont la temporalité est susceptible de s'accorder avec le projet révolutionnaire. Mais cette évolution naturelle, telle qu'elle est figurée dans le texte, 
associe un modèle darwinien (Étienne rêve à la fin du roman à l'élimination des bourgeois décadents par les prolétaires plus nombreux et mieux armés dans la lutte pour l'existence) et un modèle goethéen de la métamorphose, modèle qui n'est pas seulement scientifique mais qui configure également la structure romanesque de l'œuvre. L'antagonisme du paradigme biologique de l'évolution et du paradigme historique de la révolution se neutralise ainsi dans la logique littéraire du roman de formation.

Pour conclure, la relation entre les concepts d'évolution et de révolution chez Michelet, Flaubert et Zola montre bien comment se confrontent et s'entremêlent les représentations du temps historique et celles du temps biologique au XIX ${ }^{e}$ siècle. Mais il apparait aussi que la question des rapports entre évolution et révolution est approfondie par ces auteurs, non seulement sur un plan philosophique et théorique, mais aussi sur le plan artistique, si bien que ce questionnement pourrait apparaître comme le révélateur des poétiques spécifiques à chaque écrivain. Chez Michelet, c'est à travers une poétique de la « métamorphose ascendante $»^{73}$, selon l'expression employée par l'écrivain dans ses notes de la Mer, que se concilient évolution et révolution, histoire des espèces et histoire humaine, toutes deux travaillées par «le triomphe progressif de la liberté ${ }^{74} »$. Chez Flaubert, c'est dans le travail ironique du cliché que se construisent et se déconstruisent les concrétions conceptuelles qui agglutinent la révolution historique et l'évolution biologique. Chez Zola enfin, c'est à l'intérieur de la composition romanesque et dans l'élaboration de la métaphore que se combinent à l'intérieur d'une même temporalité vivante, le rythme continu de l'évolution et le rythme asyndétique de la révolution.

\section{NOTES}

1. Pour une histoire détaillée de ce mot, voir le livre d'Alain Rey, "Révolution », Histoire d'un mot, Paris, Gallimard, 1989.

2. Ibid., p. 79-81.

3. Georges Cuvier, "Mémoire sur les espèces d'éléphants vivants et fossiles ", Magasin encyclopédique, $2^{\mathrm{e}}$ année, 1796, t. III, p. 444.

4. William Whewell, History of the Inductive Sciences, 3 vol. , Londres, Parker, 1837.

5. Jules Michelet, La Montagne, Paris, Librairie internationale, 1868, p. 123-125.

6. Ibid., p. 370.

7. Ibid., p. 123.

8. La géologie actualiste postule que les processus géologiques passés sont identiques à ceux que l'on observe actuellement, mais contrairement à l'uniformitarisme de Lyell, n'affirme pas que ces processus sont parfaitement continus, de rythme et d'intensité invariables.

9. Jules Michelet, La Montagne, op. cit., p. 125.

10. Ibid., p. 126-127.

11. Sur la question de l'évolutionnisme de Michelet voir Edward Kaplan, «Michelet évolutionniste ", Romantisme, $\mathrm{n}^{\circ} 10$; "Michelet cent ans après ", 1975, p. 111-128; et plus particulièrement sur le rapport de Michelet à Darwin, voir les notes de Jean Borie, dans son édition de Jules Michelet, La Mer, Paris, Gallimard, « Folio classique », 1983, p. 393-394.

12. Jules Michelet, La Mer, op. cit., p. 192. 
13. Ibid., p. 191.

14. Michelet a pu se contenter du résumé proposé par la notice «Crustacé » du Dictionnaire classique d'histoire naturelle, rédigée par Victor Audouin.

15. Étienne Geoffroy Saint-Hilaire, "Considérations générales sur la vertèbre ", Mémoires du muséum d'histoire naturelle, Paris, Belin, 1822, t. IX, p. 89-119.

16. Étienne Geoffroy Saint-Hilaire, «Des insectes », Annales générales des sciences physiques, t. III, 1820, p. 165-180, p. 173.

17. Patrick Tort, La Raison classificatoire, Paris, Aubier, 1989, p. 455-456.

18. Jules Michelet, La Mer, op. cit., p. 191.

19. Ibid.

20. Gustave Flaubert, Correspondance, éd. Jean Bruneau et, pour le tome V, Jean Bruneau et Yvan Leclerc, Paris, Gallimard, "Bibliothèque de la Pléiade", 1973-2007, 5 volumes, t. V, Paris, Gallimard, «Bibliothèque de la Pléiade », 2007, p. 347 (lettre à Edma Roger des Genettes du 12 janvier 1878).

21. «Un après-midi, comme ils retournaient des silex au milieu de la grande route, M. le curé passa, et les abordant d'une voix pateline :

- » Ces messieurs s'occupent de géologie ? Fort bien!»

Car il estimait cette science. Elle confirme l'autorité des Écritures, en prouvant le Déluge.» (Gustave Flaubert, Bouvard et Pécuchet et Dictionnaire des idées reçues, édition établie par Stéphanie Dord-Crouslé, Paris, Flammarion, « GF », 2008, p. 134).

22. Ibid., p. 148.

23. Gustave Flaubert, Dictionnaire des idées reçues, op. cit., p. 435.

24. Gustave Flaubert, Correspondance, t. V, éd. cit., p. 739-740 (lettre à Maxime Du Camp du 13 novembre 1879). Pour une étude détaillée de cette controverse entre Flaubert et Maxime Du Camp, voir Stéphanie Dord Crouslé, "Le darwinisme de Flaubert ", L'Idée de "race » dans la littérature et les sciences humaines (XVIII ${ }^{e}$ XIX ${ }^{e}$ siècles), éd. S. Moussa, Paris, L'Harmattan, coll. « Histoire des Sciences Humaines ", 2003, p. 283-297.

25. Maxime Du Camp, Les Convulsions de Paris, Paris, Hachette, 4 vol. , 1878-1880, t. 4, p. 459.

26. Voir sur le motif du struggle for life et le personnage du struggleforlifeur dans la littérature fin de siècle en France, Linda Clark, Social Darwinism in France, Alabama, university of Alabama, 1984 ; mais aussi Louise Lyle, "Charles Darwin dans Le jardin des supplices ", Cahiers Octave Mirbeau, $\mathrm{n}^{\circ} 14$, 2007, p. 47-64 et du même auteur «Le Struggle for life: Contesting Balzac through Darwin in Zola, Bourget, and Barrès ", Nineteenth Century French Studies, vol. 36, $\mathrm{n}^{\circ} 3$ et 4 , printemps-été 2008, p. 305-319.

27. «Si l'on ajoute à cela la théorie de Darwin, dont ils n'ont retenu que les côtés dangereux, on arrive fatalement au combat pour l'existence qui est l'insurrection permanente, et à la sélection, qui aboutit tout droit au despotisme. Quia nominor leo!» (Maxime Du Camp, Les Convulsions de Paris, op. cit., p. 244).

28. Yvette Conry, L'Introduction du darwinisme en France au XIX $X^{e}$ siècle, Paris, Vrin, 1974.

29. Yvette Conry, "Le darwinisme social existe-t-il?", Darwin en perspective, Vrin, 1987, p. 83-106., p. 87.

30. Ernst Hæckel, Les Preuves du transformisme. Réponse à Virchow, trad. Jules Soury, Paris, Germer Baillière, 1879, p. 111.

31. Gustave Flaubert, Correspondance, éd. cit., t. IV, p. 814 (lettre à Edma Roger des Genettes du 17 juin 1874).

32. Ibid., p. 835 (lettre à Edma Roger des Genettes du 14 juillet 1874).

33. Ibid., p. 824 (lettre à George Sand du 3 juillet 1874).

34. Gustave Flaubert, Correspondance, éd. cit., t. V, p. 739-740 (lettre à Maxime Du Camp du 13 novembre 1879). 
35. Émile Zola, Germinal, Colette Becker (éd.), Paris, Librairie Générale Française, « Les Classiques de Poche », 2000 (1885), p. 206.

36. Lettre d'Émile Zola à Jacques Van Santen Kolff du 6 octobre 1889.

37. Zola, Germinal, op. cit., p. 178.

38. Ibid., p. 281.

39. Patrick Tort, commentaires et notes sur Anton Pannekoek, Darwinisme et marxisme, traduction de Hubert de Ponthière, Guy Voets et Patrick Tort, Paris, Éditions Arkhê, 2011, p. 67.

40. Émile de Laveleye, Le Socialisme contemporain, Paris, Baillère, 1883 (1881).

41. Ibid., p. 26.

42. Ibid., p. 25-26.

43. Ibid., p. XI.

44. Zola, Germinal, op. cit., p. 493.

45. Ibid., p. 327.

46. Karl Marx, Lettres sur les sciences de la nature (et les mathématiques), Karl Marx et Friedrich Engels, traduction et introduction Jean-Pierre Lefèbvre, Paris, Éditions sociales, 1974, p. 21.

47. Émile Zola, Germinal, op. cit., p. 493.

48. Paul Lafargue, Le Matérialisme économique de Karl Marx. Le milieu naturel : théorie darwinienne, Paris, H. Oriol, 1884, vol. II, p. 14.

49. Émile Zola, Germinal, op. cit., p. 282-283.

50. Émile Zola, La Fabrique des Rougon-Macquart. Édition des dossiers préparatoires. Volume V, éd. Colette Becker et Véronique Lavielle, Paris, Honoré Champion, 2011, p. 218, f ${ }^{\circ} 177$.

51. Colette Becker, « Introduction » à Germinal, op. cit., p. 26.

52. Germinal, op. cit., p. 173.

53. Ibid., p. 174.

54. Ibid., p. 163.

55. Ibid., p. 481.

56. Jean-Louis Cabanès explique ainsi que pour Zola «la conception de l'histoire est double: tantôt il l'envisage comme une suite de progrès cumulatifs, tantôt il se la représente comme ponctuée par des catastrophes [...]. La vertu du sang versé tient d'une thérapeutique de résurrection, préludant, comme les crises dans la médecine hippocratique, à un réaménagement $\mathrm{du}$ corps social.» («Un monde avec un jugement sur le monde. Éléments d'une morale zolienne ", Les Cahiers naturalistes, XLVIII, $\mathrm{n}^{\circ}$ 76, 2002, p. 363-372).

57. Émile Zola, Germinal, op. cit., p. 568.

58. Émile Zola, Germinal, op. cit., p. 179, 180 et 279.

59. La Fabrique des Rougon-Macquart. Édition des dossiers préparatoires, vol. V, op. cit., p. 110.

60. Émile Zola, Germinal, op. cit., p. 568.

61. N. a. fr. $10333 \mathrm{f}^{\circ} 496$.

62. Émile Zola, Les Quatre Évangiles : Travail, Paris, Fasquelle, 1901, p. 385.

63. Ibid.

64. Élisée Reclus, L'évolution, la révolution et l'idéal anarchiste, Paris, Stock, 1914 (1898), p. 15.

65. Ibid.

66. Émile Zola, Les Quatre Évangiles : Travail, op. cit., p. 190.

67. Émile Zola, Les Trois villes : Paris, Paris, Charpentier et Fasquelle, 1898, p. 227.

68. Émile Zola, La Fabrique des Rougon-Macquart. Édition des dossiers préparatoires. Volume V, p. 458, $\mathrm{f}$ - 394.

69. Émile Zola, Germinal, op. cit., p. 564.

70. Ibid., p. 567.

71. Ibid., p. 565.

72. Henri Mitterand affirme que «le contenu historique et social [de Germinal] est débordé par l'imagerie biologique et cosmique. Les crises de la société contemporaine y sont assimilées aux 
cataclysmes naturels qui affectent périodiquement l'ordre du monde, sans en modifier les structures profondes. »(Le Discours du roman, Paris, PUF, 1980, p. 156). Sur ce processus de naturalisation de l'historique et sur sa signification ambiguë au plan politique et idéologique voir également Sandy Petrey, « Nature et histoire au mois de Germinal ", Europe, n 678, 1985, et Nikas Bender, La lutte des paradigmes: la littérature entre histoire, biologie et médecine (Flaubert, Zola, Fontane), Amsterdam, Rodopi, 2010, p. 301-359.

73. Jules Michelet, La Mer, op. cit., p. 332, note du livre II, chap. IV, V, VI.

74. Jules Michelet, Introduction à l'histoire universelle, Euvres complètes, Paris, Flammarion, 1972, t. II, p. 229.

\section{RÉSUMÉS}

À travers l'étude de trois auteurs, Michelet, Flaubert et Zola, nous nous proposons d'étudier l'interaction de deux conceptions concurrentes du temps dans la littérature du XIX ${ }^{e}$ siècle : la temporalité évolutionniste, qui suppose la représentation d'un temps continu et progressif et la temporalité révolutionnaire, qui est liée au contraire à la vision d'un temps asyndétique et saccadé. Si la première temporalité est enracinée dans le champ de la biologie et la seconde dans celui de l'histoire et de la politique, la sociobiologie propose au $\mathrm{XIX}^{\mathrm{e}}$ siècle une vision " évolutionnaire » du devenir des sociétés, et la pensée catastrophiste d'un Cuvier conçoit l'histoire du globe sur le modèle du soubresaut révolutionnaire. Les relations complexes qu'entretiennent les concepts d'évolution et de révolution chez les auteurs du XIX ${ }^{\mathrm{e}}$ siècle sont tributaires de ce délicat nouage, propre à chaque univers littéraire, entre nature et culture, entre biologie et histoire.

\section{INDEX}

Keywords : revolution, évolution, catastrophism, historical temporality, biological temporality Mots-clés : révolution, évolution, catastrophisme, temps historique, temps biologique

\section{AUTEUR}

\section{JULIETTE AZOULAI}

Laboratoire LISAA, UPEM et ANR Biolograpes 